\title{
Pandemic triage: the ethical challenge
}

\author{
Ryan M. Melnychuk, Nuala P. Kenny
}

$\infty \quad$ See related article page I377

I $\mathrm{n}$ their article describing a triage protocol for critical care during an influenza pandemic, Christian and associates (page 1377$)^{1}$ identify some of the medical and ethical issues that are likely to arise during a pandemic. The authors have attempted to close a significant gap in the Canadian influenza pandemic plan related to triage and the prioritization and use of scarce resources, ${ }^{2}$ while also considering the ethical repercussions of such a protocol. Pandemic influenza or, as SARS demonstrated, any catastrophic outbreak of infectious disease will have profound effects on the availability and delivery of health care services and the functioning of health care institutions. As Christian and associates make explicit, in the absence of a thoughtful approach, health care resources will be depleted within the first weeks of a pandemic. The authors conclude that "development of a triage protocol is a complex process that cannot be undertaken during a pandemic." As a result, both precaution and foresight planning ${ }^{3}$ are needed to achieve the primary goals of the Canadian Pandemic Influenza Plan: "first to minimize serious illness and overall death and second to minimize societal disruption."

Christian and associates state that "under normal circumstances, all patients should have an equal claim to receive the health care they need." Here, an important distinction can be made between equity of access and equity of outcomes from available health care resources and services, both of which are critical aspects of this protocol. The first concept, equity of access, is related to the 4 primary components of the triage protocol: inclusion criteria, exclusion criteria, minimum qualifications for survival and a 4-category colour-coded prioritization tool. Since resources will be drawn from a single common pool, the protocol does not differentiate between uninfected patients and those infected with influenza. Instead, individuals who are worst off but deemed most likely to benefit from admission to an intensive care unit (ICU) and access to ICU services, on the basis of the criteria and tools listed above, will be admitted for treatment, including ventilation. Thus, the protocol, which is "aimed at maximizing benefits for the largest number of patients presenting to an overwhelmed critical care system," adheres to utilitarianism, where utility necessitates "balanc[ing] benefits and drawbacks to produce the best overall results." ${ }^{15}$ In the context of a pandemic, or during other public health emergencies, the best overall result is to minimize serious illness and death by administering a finite pool of resources to those who have the greatest opportunity to benefit from them.
The second consideration, which is directly linked to equity of access and the value of fairness invoked to defend this protocol, is equity of outcomes. Some patients will be denied access to critical care resources and will be discharged or excluded from the ICU because of their predicted inability to benefit from care; nonetheless, as a matter of fairness, these patients must be provided with appropriate palliative care or pain management, or both.

Moreover, since health and well-being demand special moral consideration, patients not only have an equal claim to the health care from which they will benefit under normal circumstances, they also have an equal claim to the health care from which they will benefit under all circumstances. Although the applicability and relative weight of values and principles may differ during emergency and non-emergency situations, these values and principles do not change: "The same basic morality applies to any human interactions. But when the same rules are applied in different contexts, different conclusions result." ${ }^{\prime}$ During a public health emergency, such as an influenza pandemic, the emphasis will shift from the individual to the population. Therefore, while caring for individual patients with specific needs, health care workers must also consider communities, the public interest and the common good.

Stand on Guard for Thee, ${ }^{7}$ a document produced by the Pandemic Influenza Working Group at the University of Toronto's Joint Centre for Bioethics, is cited as the primary ethical foundation for this protocol. Yet it is unclear how the substantive principles in that document shaped the protocol, since Christian and associates refer only to proportionality. Specifically, we are puzzled as to how their assessment of the other substantive values listed in Stand on Guard for Thee - individual liberty, protection of the public from harm, privacy, duty to care, reciprocity, equity, trust, solidarity and stewardship — resulted in a classic utilitarian basis for the protocol. It is precisely in the weighing of these principles that the hard work of ethics is done, since the framework of the working group's document does not include a mechanism to weigh and specify principles.

Christian and associates acknowledge that this protocol has never been employed and that it was developed by a panel of experts, without public input. They also note that engaging the community and the greater public in the foundational values of this protocol will be of great importance, but unfortunately they do not address how this will be accomplished. Openness or transparency, a crucial step toward building the necessary public trust, is a procedural value that is rightly identified in 
Stand on Guard for Thee; however, without a carefully contemplated strategy of public engagement, public education and trust may be difficult to capture when we need it most.

In the absence of a national ethics framework, Christian and associates have addressed one of many difficult decisions that will confront pandemic planners across Canada, and they are to be commended for their effort. However, their article is missing a crucial element: they provide a robust scientific discussion of triage, stating that a set of substantive and procedural values informed their triage protocol, but they do not tell us exactly how that occurred. There is an urgent need for a national ethical framework that makes explicit the values and principles that will guide pandemic influenza planning. The development of such a framework entails real work in the formulation of policy and its implementation; we must be clear why certain values are privileged and others not. This paper contributes to the project, but there is much tough work yet to be done. Three key interconnected values that are identified in Stand on Guard for Thee appropriately focus on the common good and thus are highly relevant to pandemic planning: equity (we need to maintain equity and not increase inequity in the face of fear and uncertainty), trust (we need to have trust in both fair processes and fair treatments) and solidarity (we are all in this together, and protecting the public and hence ourselves will require society-wide collaborations). By maintaining equity, building trust and promoting solidarity, the common good can be more effectively promoted and protected.

This article has been peer reviewed.

Ryan Melnychuk is a Postdoctoral Fellow in Ethics of Health Research and Policy with the Department of Bioethics, Dalhousie University, and a Post-Doctoral Fellow in the Health Policy and Translation Research Group with the Canadian Center for Vaccinology, IWK Health Centre, Halifax, NS. Nuala Kenny is a Professor with the Department of Bioethics, Dalhousie University, Halifax, NS.

Competing interests: None declared.

Acknowledgement: Ryan Melnychuk is supported by a Canadian Institutes of Health Research training grant in Ethics of Health Research and Policy.

\section{REFERENCES}

I. Christian MD, Hawryluck L, Wax RS, et al. Development of a triage protocol for critical care during an influenza pandemic. $C M A J$ 2006;175(II):1377-8I.

2. Kotalik J. Preparing for an influenza pandemic: ethical issues. Bioethics 2005; I9:422-3I.

3. Morris J. Rethinking risk and the precautionary principle. Oxford (UK): Butterworth-Heinemann; 2000. p. I.

4. National Pandemic Influenza Committee. Canadian pandemic influenza plan. Ottawa: Health Canada; 2004. p. 3. Available: www.phac-aspc.gc.ca/cpip-pclcpi /pdf-cpip-03/canadian-pandemic-influenza-plan.pdf (accessed 2006 Oct 12).

5. Beauchamp TL, Childress JF. Principles of biomedical ethics. 5 th ed. New York: Oxford University Press; 200I. p. I65.

6. Gert HJ. How are emergencies different from other medical situations? Mt Sinai J Med 2005;72:216-20.

7. University of Toronto Joint Centre for Bioethics Pandemic Influenza Working Group. Stand on guard for thee: ethical considerations in preparedness planning for pandemic influenza. Toronto: University of Toronto Joint Centre for Bioethics; 2005. Available: www.utoronto.ca/jcb/home/documents/pandemic.pdf (accessed 2006 Oct I2).

Correspondence to: Dr. Ryan M. Melnychuk, Rm. C315, CRC Building, Department of Bioethics, Dalhousie University, 5849 University Ave., Halifax NS $\mathrm{B}_{3} \mathrm{H}_{4} \mathrm{H}_{7}$; fax 902 494-3865; ryan.melnychuk@dal.ca 